\title{
PLANE CUBICS AND IRRATIONAL COVARIANT CUBICS*
}

\author{
BY \\ HENRY S. WHÌTE
}

\section{Introduction.}

Every covariant containing two contragredient sets of variables, of the same degree in both sets of variables, may be interpreted as the symbol of a transformation. For the lineo-linear form indeed this is the most common interpretation, a collineation; and the symbol of such a transformation is applied to a form or quantic of order $n$ as many times simultaneously as there are units in its order, $n$. Thus if the transformation be denoted by the lineo-linear form $a_{x} u_{a}$, and the quantic of the third order by $A_{x}^{3}$, the transformed cubic is denoted by

$$
A_{a} A_{a^{\prime}} A_{a^{\prime \prime}} a_{x} a_{x}^{\prime} a_{x}^{\prime \prime} \equiv A_{x}^{\prime 3} .
$$

Algebraically, however, we might describe this as the complete transformation; and no less interest would attach to the partial transformations, whose effect would be denoted by

$$
A_{a} A_{x}^{2} a_{x}=A_{x}^{\prime 3}, \quad A_{a}^{\prime} A_{x}^{\prime 2} a_{x}=A_{x}^{\prime \prime 3} \text {, etc. }
$$

Similarly those mixed covariants of a ternary cubic which have their class and order equal may be applied: first, to effect complete transformation upon quantics of their own order or class; second, to effect partial transformation upon quantics of an order or class higher than their own. Employed in this way, they lead to the study of curves which are unchanged by such a transformation. These curves are properly described as curves irrationally covariant to the fundamental cubic.

In the theory of the ternary cubic there are seven mixed covariants of the specified sort, three of order and class $(2,2)$, and four of order and class $(3,3)$. Conics invariant under complete transformation of two of the three kinds $(2,2)$ have been discussed in a paper published in the preceding number of the Transactions. In the present paper it is proposed to consider cubics invariant under partial transformation by covariants $(2,2)$, and those invariant.

* Presented to the Society (Chicago) December 29, 1899. Received for publication February $15,1900$. 
under complete transformation by covariants $(3,3)$. There will remain for subsequent treatment the two sets of conics invariant under the third transformation $(2,2)$, and invariant curves of order higher than the third. Both these topics present points of interest and novelty.

The new covariant cubics found by this investigation are eight in number, all of the type called equianharmonics. Dually of course there are found eight corresponding curves of class three. In conclusion are discussed the relations of apolarity in these two sets, and the relation of each set to the four inflexional triangles.

\section{§1. Cubics connected with Covariant Conics.}

The ternary cubic has three linearly independent covariants of order 2 and class 2. These are denoted in Clebsch's and Gordan's writings by $\Theta, \mathbf{H}$, and $\mathrm{K}$. If . we write $a_{x}^{3}$ for the fundamental cubic and $a_{x}^{3}$ for its Hessian, then these three have the symbolic structure :

$$
\begin{aligned}
& \Theta=\left(a a^{\prime} u\right)^{2} a_{x} a_{x}^{\prime}=\Theta_{x}^{2} u_{\vartheta}^{2}, \\
& \mathrm{H}=(a a u)^{2} a_{x} a_{x}=\mathrm{H}_{x}^{2} u_{\eta}^{2}, \\
& \mathrm{~K}=\left(a a^{\prime} u\right)^{2} a_{x} a_{x}^{\prime}=\mathrm{K}_{x}^{2} u_{\kappa}^{2} .
\end{aligned}
$$

In the former paper I have employed $\Theta$ as a symbol of operation, and have discussed the conics that are transformed into themselves by that operation. Considering it first as a transformer of point loci, second as a transformer of lineloci, we found in the one case two nets, in the other two webs of conics which were termed respectively autopolo-conics and conic autopolars. These were the polar systems of two pairs of cubies, termed autopolo-cubics and cubic autopolars. Are there also other autopolo-cubics, and how are they defined geometrically? Neglecting, temporarily, the dual aspect of the transformers, we extend the inquiry also to point-loci of third order invariant to transformation by $\mathrm{H}$ and $\mathrm{K}$ respectively. Of these, two in each case have for polar-systems nets of conics invariant with respect to the same transformation. While these have peculiarly interesting features, we shall postpone their discussion and limit our inquiry to cubics whose conic polars are not invariant with respect to any rational covariant transformation of order and class lower than 3 ; such may be termed special covariant cubics.

\section{\$2. Special Covariant Cubics whose Transformers are Reducible.}

As transformers of point loci consider three reducible forms $\Theta_{x}^{2} u_{\vartheta}^{2} u_{x}, \mathrm{H}_{x}^{2} u_{\eta}^{2} u_{x}$, and $\mathrm{K}_{x}^{2} u_{\kappa}^{2} u_{x}$. Call them $\Theta, \mathrm{H}, \mathrm{K}$, as before, remembering that they are now 
extended so as to be of order and class 3. Define them for use as transformers thus, the ternary cubic $L_{x}^{3}$ being indeterminate :

$$
\begin{aligned}
\Theta\left(L_{x}^{3}\right) & =\Theta_{x}^{2} L_{\vartheta}^{2} L_{x}, \\
\mathrm{H}\left(L_{x}^{3}\right) & =\mathrm{H}_{x}^{2} L_{\eta}^{2} L_{x}, \\
\mathrm{~K}\left(L_{x}^{3}\right) & =\mathrm{K}_{x}^{2} L_{\kappa}^{2} L_{x} .
\end{aligned}
$$

We are to determine cubics $L_{x}^{3}$ satisfying the condition of invariantiveness with respect to each of these transformations in turn.

First : the operator $\Theta$.

We impose upon $L_{x}^{3}$ the condition :

$$
\Theta\left(L_{x}^{3}\right)=\Theta_{x}^{2} L_{\vartheta}^{2} L_{x} \equiv \lambda \cdot L_{x}^{3} .
$$

The fundamental cubic being taken in the canonical form of Hesse :

$$
a_{x}^{3}=x_{1}^{3}+x_{2}^{3}+x_{3}^{3}+6 m x_{1} x_{2} x_{3},
$$

this identity is equivalent to the following ten equations :

$$
\begin{aligned}
& 2 m^{2} L_{111}+4 m L_{123}=\lambda \cdot L_{111}, \\
& 2 m^{2} L_{222}+4 m L_{123}=\lambda \cdot L_{222}, \\
& 2 m^{2} L_{333}+4 m L_{123}=\lambda \cdot L_{333}, \\
& 2\left(L_{111}+L_{222}+L_{333}\right)+12 m^{2} L_{123}=-6 \lambda \cdot L_{123} ; \\
& \quad-2 m^{2} L_{112}+4 m L_{223}-2 L_{133}=3 \lambda \cdot L_{112}, \\
& \quad-2 m^{2} L_{223}+4 m L_{133}-2 L_{112}=3 \lambda \cdot L_{223}, \\
& \quad-2 m^{2} L_{133}+4 m L_{112}-2 L_{223}=3 \lambda \cdot L_{133} ; \\
& -2 m^{2} L_{122}+4 m L_{113}-2 L_{233}=3 \lambda \cdot L_{122}, \\
& -2 m^{2} L_{233}+4 m L_{122}-2 L_{113}=3 \lambda \cdot L_{233}, \\
& -2 m^{2} L_{113}+4 m L_{233}-2 L_{122}=3 \lambda \cdot L_{113} .
\end{aligned}
$$

We seek a solution $\left(L_{111}, \cdots, L_{333}, \lambda\right)$ in which not all the coefficients $L_{i j k}$ are 0 . The ten coefficients enter, four in the equations $\mathrm{I}$, three in the equations II, and three in the equations III. Unless all its coefficients $L$ are 0 each group of equations determines values of $\lambda$. The groups II and III evidently determine in this way the same three values of $\lambda$; these values are distinct from those determined by group I. 
Ad I) Unless $L_{123}=0$, it appears from inspection of equations (I) that

$$
L_{111}=L_{222}=L_{333}
$$

and that $\lambda$ is a root of the quadratic equation:

$$
\lambda^{2}=4\left(m^{4}-m\right)=\frac{1}{6} S,
$$

$S$ denoting the well-known quartic invariant of the fundamental cubic. To find the equation $L_{x}^{3}=0$ corresponding to these two discrete values of $\lambda$, let

$$
\frac{L_{123}}{L_{111}}=\frac{L_{123}}{L_{222}}=\frac{L_{123}}{L_{333}}=L
$$

and eliminate $\lambda$ from the fourth and any other of equations (I). The result:

$$
4 m L(m+L)+1=0,
$$

is the equation found in the former paper* characterizing the two cubics ( $A$ and $B$ ) which have the same Cayleyan curve with the fundamental cubic, and whose conic polars all are invariant to the transformer $\Theta$.

For special autopolo-cubics, therefore, we must examine the remaining (eight) values of $\lambda$.

If $L_{123}=0$, we have from group (I):

$$
\lambda=2 m^{2}, \quad L_{111}+L_{222}+L_{333}=0:
$$

and the corresponding sheaf of cubics has the equation :

$$
\left(c_{2}-c_{3}\right) x_{1}^{3}+\left(c_{3}-c_{1}\right) x_{2}^{3}+\left(c_{1}-c_{2}\right) x_{3}^{3}=0,
$$

where $c_{1}, c_{2}$, and $c_{3}$ are arbitrary parameters. The discussion of this sheaf of cubics may be postponed until the equations (II) and (III) have been examined.

$A d$ II) From the cyclic character of group (II) it is seen that the following sets of values will satisfy these conditions :

$$
\begin{array}{ll}
L_{112}=L_{223}=L_{331}, & \lambda=-\frac{2}{3} \cdot(m-1)^{2} ; \\
L_{112}=\omega^{2} L_{223}=\omega L_{331}, & \lambda=-\frac{2}{3} \cdot(m-\omega)^{2} ; \\
L_{112}=\omega L_{223}=\omega^{2} L_{331}, & \lambda=-\frac{2}{3} \cdot\left(m-\omega^{2}\right)^{2} .
\end{array}
$$

Here $\omega$ and $\omega^{2}$ denote the imaginary cube roots of unity.

$A d$ III) Similarly the solutions of group (III) are found to be the following :

\footnotetext{
* loc. cit., p. 3, formulas (7) and (9).
} 


$$
\begin{array}{ll}
L_{122}=L_{233}=L_{311}, & \lambda=-\frac{2}{3} \cdot(m-1)^{2} ; \\
L_{122}=\omega^{2} L_{233}=\omega L_{311}, & \lambda=-\frac{2}{3} \cdot(m-\omega)^{2} ; \\
L_{122}=\omega L_{233}=\omega^{2} L_{311}, . & \lambda=-\frac{2}{3} \cdot\left(m-\omega^{2}\right)^{2} .
\end{array}
$$

Accordingly the roots $\lambda$ of (II) and (III) are equal in pairs, and there is one pair of equal values $\lambda$ in group (I). These pairs of equal roots indicate four singly infinite sheaves of autopolo-cubics, namely the following:

$$
\begin{array}{rr}
c_{1}\left(x_{1}^{2} x_{2}+x_{2}^{2} x_{3}+x_{3}^{2} x_{1}\right)+c_{2}\left(x_{1} x_{2}^{2}+x_{2} x_{3}^{2}+x_{3} x_{1}^{2}\right)=0, & \\
c_{1}\left(x_{1}^{2} x_{2}+\omega x_{2}^{2} x_{3}+\omega^{2} x_{3}^{2} x_{1}\right)+c_{2}\left(x_{1} x_{2}^{2}+\omega x_{2} x_{3}^{2}+\omega^{2} x_{3} x_{1}^{2}\right)=0, & {\left[\lambda=-\frac{2}{3}(m-1)^{2}\right] ;} \\
c_{1}\left(x_{1}^{2} x_{2}+\omega^{2} x_{2}^{2} x_{3}+\omega x_{3}^{2} x_{1}\right)+c_{2}\left(x_{1} x_{2}^{2}+\omega^{2} x_{2} x_{3}^{2}+\omega x_{3} x_{1}^{2}\right)=0, & {\left[\lambda=-\frac{2}{3}(m-\omega)^{2}\right] ;} \\
\left(c_{2}-c_{3}\right) x_{1}^{3}+\left(c_{3}-c_{1}\right) x_{2}^{3}+\left(c_{1}-c_{2}\right) x_{3}^{3}=0, & {\left[\lambda=-\frac{2}{3}\left(m-\omega^{2}\right)^{2}\right] ;}
\end{array}
$$

The autopolo-cubics of a non-singular plane cubic are the two discrete cubics whose conic polars are autopolo-conics of the fundamental curve, and four sheaves of special covariant cubics, all equianharmonic, whose equations for the canonical form are written as $(9 a, 9 b, 9 c, 4 d)$ above.

Second: the operator $\mathrm{K}$.

The discussion of the operator $\Theta$ may be repeated for the operator $K$ with no other change than the substitution of $-\left(1+2 \mathrm{~m}^{3}\right) / 6 \mathrm{~m}^{2}$, the parameter of the Hessian, in place of $m$, the parameter of the fundamental cubic. The values of $\lambda$ compatible with the condition:

$$
\mathrm{K}_{x}^{2} L_{\kappa}^{2} L_{x}=\lambda \cdot L_{x}^{3},
$$

are accordingly the two discrete roots of the quadratic equation :

$$
\lambda^{2}=\frac{1}{\overline{7}}\left(8 T^{2}-S^{3}\right),
$$

and the following four, each counted doubly :

$$
\begin{aligned}
& \lambda=-\frac{2}{3}\left(1-6 m^{2}+2 m^{3}\right)^{2}, \\
& \lambda=-\frac{2}{3}\left(1-6 \omega m^{2}+2 m^{3}\right)^{2}, \\
& \lambda=-\frac{2}{3}\left(1-6 \omega^{2} m^{2}+2 m^{3}\right)^{2}, \\
& \lambda=2\left(1+2 m^{3}\right)^{2} .
\end{aligned}
$$

The two discrete roots will give two cubics of the syzygetic sheaf, non-special, different from those found by the operator $\Theta$; but the four sheaves of cubics 
corresponding to the remaining roots are the same for both operators, since their equations $(9 a, 9 b, 9 c, 9 d)$ are independent of the parameter $m$; while the fundamental cubic and its Hessian are reduced to Hesse's canonical form simultaneously and $\mathrm{K}$ is the operator $\Theta$ formed from the Hessian instead of the fundamental cubic.

The cubics invariant with respect to the operator $\mathrm{K}$ are the autopolocubics of the Hessian, and consist of two discrete curves whose conic polars are the autopolo-conics of the Hessian, together with the same,four simply infinite sheaves of cubics that are invariant with respect to the operator $\Theta$.

Third: the operator $\mathrm{H}$.

Expanding for the canonical form of the cubic the required identity:

$$
\mathrm{H}_{x}^{2} L_{\eta}^{2} L_{x} \equiv \lambda \cdot L_{x}^{3}
$$

we have ten equations elosely resembling groups (I), (II), (III) under the operator $\Theta$. From four typical examples the entire set may be obtained by permutation of indices :

$$
\begin{array}{cc}
2 m \mu L_{111}+2(m+\mu) L_{123}=\lambda \cdot L_{111}, & (3 \text { equations); } \\
-2\left(L_{111}+L_{222}+L_{333}\right)-12 m \mu L_{123}=6 \lambda L_{123}, & \text { (1 equation); } \\
-2 m \mu L_{112}+2(m+\mu) L_{223}-2 L_{133}=3 \lambda L_{112}, & \text { (3 equations); } \\
-2 m \mu L_{113}+2(m+\mu) L_{233}-2 L_{122}=3 \lambda L_{113}, & \text { (3 equations); }
\end{array}
$$

$\mu$ denoting the parameter of the Hessian.

$A d$ IV) Equating to zero as before all coefficients $L_{i j k}$ which do not occur in those equations, for otherwise none of the others yield the same values of $\lambda$, we find two solutions which are not special, and two equal roots $\lambda$ indicating a sheaf of special cubics. The former are found by assuming

whence

$$
L_{123} \neq 0 \text {, }
$$

$$
\begin{aligned}
& \lambda= \pm 4 m\left(1-m^{3}\right), \\
& L_{111}=L_{222}=L_{333} ;
\end{aligned}
$$

then if we set

we have the relation:

$$
\frac{L_{123}}{L_{111}}=L
$$

$$
1+4 m \mu \cdot L+2(m+\mu) \cdot L^{2}=0 .
$$

This relation is exactly that which gives the parameters of two nets of conics 
invariant with respect to the transformation $\mathrm{H}$, the polar nets of the cubics here found. These therefore are not special covariant cubies.

Special cubics are found by taking

whence

$$
L_{123}=0 \text {, }
$$

$$
\begin{gathered}
L_{111}+L_{222}+L_{333}=0, \\
\lambda=2 m \mu=-2 m\left(1+2 m^{3}\right) .
\end{gathered}
$$

The corresponding sheaf of cubics must have the following equations, identical with $(9 d)$ :

$$
\left(c_{2}-c_{3}\right) x_{1}^{3}+\left(c_{3}-c_{1}\right) x_{2}^{3}+\left(c_{1}-c_{2}\right) x_{3}^{3}=0 .
$$

$A d \mathrm{~V}, \mathrm{VI})$ From the cyclic structure of equations $\mathrm{V}$ and VI it is evident that they have as solutions the same sheaves of cubics that were found from II and III under the operation $\Theta$. The values of $\lambda$ are determined by inspection of the determinantal equation :

$$
\begin{gathered}
\left|\begin{array}{ccc}
2 m \mu+3 \lambda & -2(m+\mu) & 2 \\
2 & 2 m \mu+3 \lambda & -2(m+\mu) \\
-2(m+\mu) & 2 & 2 m \mu+3 \lambda
\end{array}\right|=0 ; \\
\lambda=\frac{1}{3}\{2(m+\mu)-2 m \mu-2\}, \\
\lambda=\frac{1}{3}\left\{2(m+\mu) \omega-2 m \mu-2 \omega^{2}\right\}, \\
\lambda=\frac{1}{3}\left\{2(m+\mu) \omega^{2}-2 m \mu-2 \omega_{j} .\right.
\end{gathered}
$$

Aside from two discrete cubics, which are not special covariants, the cubics invariant with respect to the transformation $\mathrm{H}$ are four sheaves, identical with those left invariant by transformations $\Theta$ and $\mathrm{K}$.

\section{§3. Special Covariant Cubics whose Transformers are Irreducible.}

Mixed concomitants of order 3 and class 3 are four in number, beside the three reducible ones already discussed. Let each be denoted by $G_{i}$, where the suffix indicates the degree in the coefficients of the fundamental cubic. These four are readily calculated for the canonical form, from the fact that each is a Jacobian of simpler concomitants. Thus we may write :

$$
\begin{aligned}
& G_{3}=J\left(\Theta, f, u_{x}\right)=6(a c u)(a b u)^{2} b_{x} c_{x}^{2}, \\
& G_{5}^{\vee}=J\left(\mathrm{H}, f, u_{x}\right)=6(a b u) a_{x} b_{x}^{2} a_{s} u_{s}^{2}, \\
& G_{7}=J\left(\mathrm{~K}, f, u_{x}\right), \\
& G_{9}=J\left(\mathrm{~K}, \Sigma, u_{x}\right),
\end{aligned}
$$


the Jacobian being formed in this last case by differentiating with respect to the variables $\left(u_{1}, u_{2}, u_{3}\right)$ instead of $\left(x_{1}, x_{2}, x_{3}\right)$.

The manner of employing these to transform point-loci is already explained; e. g., we define

$$
G_{3}\left(L_{x}^{3}\right)=(a c L)(a b L)^{2} b_{x} c_{x}^{2}, \text { etc. }
$$

The calculations involved in determining cubics invariant under these four transformations are so extensive that I will present here only those for the one of lowest degree, $G_{3}$. The omission of the others may be warranted also by this noteworthy fact :

The cubics which are invariant with respect to the transformers $G_{5}, G_{7}$, and $G_{9}$ respectively are identical with those which are invariant with respect to $G_{3}$, and consist of 8 discrete cubics and a sheaf, the syzygetic sheaf of the fundamental cubic and its Hessian.

The operator $G_{3}$.

Denoting by $L_{x}^{3}$ the cubic to be determined, and by $\lambda$ an invariant factor, we impose the condition :

$$
G_{3}\left(L_{x}^{3}\right) \equiv 6 \lambda \cdot L_{x}^{3}
$$

The cubic being in the canonical form, we write the operator $G_{3}$ as in (17), when this condition becomes :

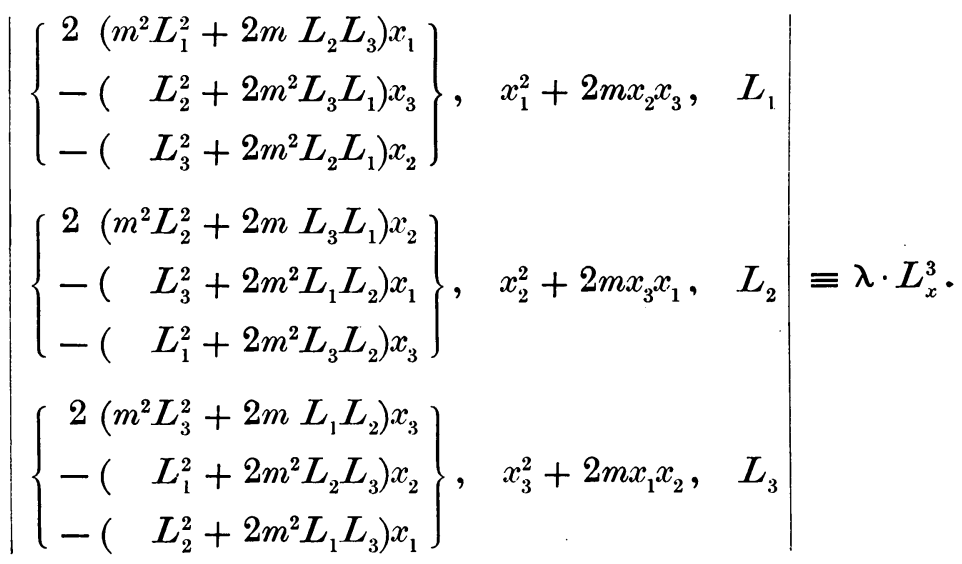

Expanding this, we find the ten conditions, given typically by the following four :

$$
\begin{array}{cc}
-L_{222}+L_{333}=\lambda \cdot L_{111}, & \text { (3 equations); } \\
0=6 \lambda \cdot L_{123}, & \text { (1 equation); } \\
-\left(1+8 m^{3}\right) L_{112}-12 m^{2} L_{223}-6 m L_{331}=3 \lambda \cdot L_{112}, & \text { (3 equations); } \\
\left(1+8 m^{3}\right) L_{122}+12 m^{2} L_{311}+6 m L_{233}=3 \lambda \cdot L_{122}, & \text { (3 equations). }
\end{array}
$$


From (VII) we find by inspection the solution $\lambda=0, L_{111}=L_{222}=L_{333}$; and combination gives the other values:

$$
\lambda= \pm \sqrt{-3} .
$$

The root $\lambda=0$ occurs also in (VIII), so that we have a simply infinite sheaf:

$$
c_{1}\left(x_{1}^{3}+x_{2}^{3}+x_{3}^{3}\right)+c_{2} \cdot x_{1} x_{2} x_{3}=0,
$$

the syzygetic sheaf of the fundamental cubic and its Hessian. The other two roots of (VII) give the two cubics :

$$
\begin{array}{ll}
x_{1}^{3}+\omega x_{2}^{3}+\omega^{2} x_{3}^{3}=0, & \left(\lambda=-\omega+\omega^{2}\right) ; \\
x_{1}^{3}+\omega^{2} x_{2}^{3}+\omega x_{3}^{3}=0, & \left(\lambda=-\omega^{2}+\omega\right) ;
\end{array}
$$

where $\omega$ is an imaginary cube root of unity.

Equations (IX) and (X) lead to the solutions, arranged alternately,

$$
\begin{aligned}
& x_{1}^{2} x_{2}+x_{2}^{2} x_{3}+x_{3}^{2} x_{1}=0, \quad \cdot \quad\left(\lambda=-\frac{1}{3}[1+2 m]^{3}\right) ; \\
& x_{1} x_{2}^{2}+x_{2} x_{3}^{2}+x_{3} x_{1}^{2}=0, \quad\left(\lambda=\frac{1}{3}[1+2 m]^{3}\right) \text {; } \\
& x_{1}^{2} x_{2}+\omega x_{2}^{2} x_{3}+\omega^{2} x_{3}^{2} x_{1}=0, \quad\left(\lambda=-\frac{1}{3}\left[1+2 \omega^{2} m\right]^{3}\right) ; \\
& x_{1}^{2} x_{3}+\omega x_{2}^{2} x_{1}+\omega^{2} x_{3}^{2} x_{2}=0, \quad\left(\lambda=\frac{1}{3}\left[1+2 \omega^{2} m\right]^{3}\right) \text {; } \\
& x_{1}^{2} x_{2}+\omega^{2} x_{2}^{2} x_{3}+\omega x_{3}^{2} x_{1}=0, \quad\left(\lambda=-\frac{1}{3}[1+2 \omega m]^{p}\right) \text {; } \\
& x_{1}^{2} x_{3}+\omega^{2} x_{2}^{2} x_{1}+\omega x_{3}^{2} x_{2}=0, \quad\left(\lambda=\frac{1}{3}[1+2 \omega m]^{3}\right) \text {. }
\end{aligned}
$$

For the transformations by $G_{5}, G_{7}$, and $G_{9}$, these invariant cubics reappear although the values of the invariant factors $\lambda$ are of course altered, in so far as they are functions of the parameter $m$ * $^{*}$ For $G_{5}$, for example, they are the following :

* For the sake of completeness I add the typical equations from which are determined the cubics invariant under the transformations symbolized by the mixed covariants $G_{7}$ and $G_{9}$ of degrees 7 and 9 in the coefficients of the fundamental cubic. It is understood that each set of ten equations is obtained from each of these sets of four by cyclical permutation of the indices $1,2,3$.

From the condition : $G_{7}\left(L_{x}^{3}\right)=\lambda \cdot L_{x}^{3}$, one has the equations :

$$
\begin{aligned}
\lambda L_{1}^{3} & =-L_{2}^{3}+L_{3}^{3}, \\
-3 \lambda L_{1}^{2} L_{2} & =\left(1+8 m \mu^{2}\right) L_{1}^{2} L_{2}+4 \mu(2 m+\mu) L_{2}^{2} L_{3}+2(m+2 \mu) L_{3}^{2} L_{1}, \\
3 \lambda L_{1} L_{2}^{2} & =\left(1+8 m \mu^{2}\right) L_{1} L_{2}^{2}+4 \mu(2 m+\mu) L_{3} L_{1}^{2}+2(m+2 \mu) L_{2} L_{3}^{2}, \\
6 \lambda L_{1} L_{2} L_{3} & =0 .
\end{aligned}
$$

Here $\mu$ denotes the parameter of the Hessian.

From the condition : $G_{9}\left(L_{x}^{3}\right)=\lambda L_{x}^{3}$, one has the equations :

$$
\begin{array}{rlrl}
\lambda L_{1}^{3}= & -3 m^{3}\left(5+4 m^{3}\right)\left(L_{2}^{3}-L_{3}^{3}\right), \\
3 \lambda L_{1}^{2} L_{2}= & \left(1-m^{3}\right)\left(1+4 m^{3}\right)\left(1+8 m^{3}\right) L_{1}^{2} L_{2}+m\left(6+5 m-60 m^{2}-16 m^{4}-16 m^{7}\right) L_{2}^{2} L_{3}, \\
-3 \lambda L_{1} L_{2}^{2}= & \left(1-m^{3}\right)\left(1+4 m^{3}\right)\left(1+8 m^{3}\right) L_{1} L_{2}^{2} & +\left(2+3 m-20 m^{2}-8 m^{3}+80 m^{5}+24 m^{\top}\right) L_{3}^{2} L_{1}, \\
6 \lambda L_{1} L_{2} L_{3}= & \left.0.60 m^{2}-16 m^{4}-16 m^{7}\right) L_{3} L_{1}^{2}
\end{array}
$$




$$
\begin{array}{ll}
\text { (a) } & \lambda=0, \\
\left(b_{1}\right) & \lambda=-\omega+\omega^{2},
\end{array}
$$$$
\left(b_{2}\right) \quad \lambda=-\omega^{2}+\omega,
$$

$\left(c_{1}, c_{2}\right) \quad 3 \lambda=\mp\left[\left(1+8 m^{2} \mu\right)+4 m(2 \mu+m)+2(\mu+2 m)\right]$,

$\left(d_{1}, d_{2}\right) \quad 3 \lambda=\mp\left[\left(1+8 m^{2} \mu\right)+4 \omega m(2 \mu+m)+2 \omega^{2}(\mu+2 m)\right]$,

$\left(e_{1}, e_{2}\right) \quad 3 \lambda=\mp\left[\left(1+8 m^{2} \mu\right)+4 \omega^{2} m(2 \mu+m)+2 \omega(\mu+2 m)\right]$,

( $\mu$ denoting the parameter of the Hessian).

It will be observed that these 8 cubics are particular cases of those found in sheaves as invariants of the transformations $\Theta, \mathrm{K}, \mathrm{H}$, formulas $(9 a, 9 b, 9 c, 9 d)$. They are therefore invariants of every transformation whose symbol is compounded linearly from these seven: $\Theta, \mathrm{K}, \mathrm{H}, G_{3}, G_{5}, G_{7}, G_{9}$. In other words, they are irrational combinants of the cubic and its Hessian.

\section{§4. Geometrical Relations of the Irrational Covariant Cubics.}

For the canonical form of the cubic the triangle of reference is any one of the four inflexional triangles. The vertices of the inflexional triangles have accordingly the coördinates : *

$$
\begin{aligned}
& \left(\begin{array}{lll}
1 & 0 & 0
\end{array}\right)
\end{aligned}
$$

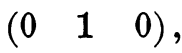

$$
\begin{aligned}
& \left(\begin{array}{lll}
0 & 0 & 1
\end{array}\right) \\
& \left(\begin{array}{lll}
1 & 1 & \omega
\end{array}\right) \\
& \left(\begin{array}{lll}
1 & \omega & 1
\end{array}\right) \text {, } \\
& \left(\begin{array}{lll}
\omega & 1 & 1
\end{array}\right)
\end{aligned}
$$

$$
\begin{aligned}
& \left(\begin{array}{lll}
1 & 1 & 1
\end{array}\right) \\
& \left(\begin{array}{lll}
1 & \omega & \omega^{2}
\end{array}\right) \text {, } \\
& \left(\begin{array}{lll}
1 & \omega^{2} & \omega
\end{array}\right) \\
& \left(\begin{array}{lll}
1 & 1 & \omega^{2}
\end{array}\right) \\
& \left(\begin{array}{lll}
1 & \omega^{2} & 1
\end{array}\right) \text {. } \\
& \left(\begin{array}{lll}
\omega^{2} & 1 & 1
\end{array}\right)
\end{aligned}
$$

Inspection shows that each of the six cubics $c_{1}, c_{2}, d_{1}, d_{2}, e_{1}, e_{2}$, contains the three vertices of the inflexional triangle $(I)$, and is tangent at each vertex to some one of its sides. The curves $b_{1}$ and $b_{2}$ do not contain those vertices, but do contain those of triangles (II), (III), and (IV). Further, the Hessian of $b_{1}$ and also of $b_{2}$ is the curve $x_{1} x_{2} x_{3}=0$, i. e., it is coincident with the triangle (I). Similar observations may be made upon the curves $c_{1}, c_{2}$ and triangle (II), the curves $d_{1}, d_{2}$ and triangle (III), and the curves $e_{1}, e_{2}$ and triangle (IV). Or the argument from symmetry may follow the first observations, giving us the theorem :

* See Clebsch-Lindemann, Vorlesungen über Geometrie, vol. I, p. 512. 
Of the eight discrete covariant cubics which are invariant with respect to the seven transformers of point-loci here considered, each one is simultaneously inscribed and circumscribed to each of three of the inflexional triangles of the fundamental cubic, and has the fourth inflexional triangle for its Hessian. *

Since these covariant cubics are all equianharmonic, it would be an interesting problem to determine how many of the systems of three triangles which can be all simultaneously inscribed and circumscribed to an equianharmonic cubic can be inflexional triangles to another cubic curve.

\section{§5. 'The Seven Corresponding Transformers of Line Loci or Envelopes.}

A single general theorem will enable us to dispense with the detailed discussion of these seven operators in the dual rôle. Consider any operator of class 3 and order $3, R_{x}^{3} u_{\rho}^{3}=R$. Employ it first as a transformer of point-loci, and denote the solutions of the invariant condition :

$$
R_{x}^{3} L_{\rho}^{3} \equiv \mu \cdot L_{x}^{3}
$$

by $L_{1}, L_{2}, \ldots$, with factors $\mu_{1}, \mu_{2}, \ldots$ Next use it to transform lineloci, and denote the solutions of the defining equation :

$$
R_{\lambda}^{3} u_{\rho}^{3} \equiv \nu \cdot u_{\lambda}^{3}
$$

by $\lambda_{1}, \lambda_{2}, \ldots$, with factors $\nu_{1}, \nu_{2}, \ldots$. If $\mu_{i}$ is different from $\nu_{\kappa}$, then $L_{i}$ is apolar to $\lambda_{\kappa}$. The proof is this : since

it follows that

$$
R_{x}^{3} L_{\rho}^{3} \equiv \mu L_{x}^{3}
$$

and since

$$
R_{\lambda}^{3} L_{\rho}^{3} \equiv \mu \cdot L_{\lambda}^{3}
$$

it follows that

$$
R_{\lambda}^{3} u_{\rho}^{3} \equiv \nu \cdot u_{\lambda}^{3}
$$

$$
R_{\lambda}^{3} L_{\rho}^{3} \equiv \nu \cdot L_{\lambda}^{3}
$$

whence by comparison, since $\mu \neq \nu$,

$$
L_{\lambda}^{3}=0
$$

and this is the expression for simple apolarity.

For each of the seven operators used, its matrical equation as a transformer of point-loci is identical with its matrical equation as a transformer of line-loci ;

* How these same cubics may be found by considering the 18 collineations which leave unaltered the fundamental cubic may be seen from a paper in the Bulletin of the American Mathematical Society, vol. 4 (1897), p. 72. 
the matrices are mutually transverse, and have the same set of roots, of which eight are discrete in the case of the irreducible cubic transformers. Hence we may draw the following conclusion :

The eight curves of class 3 which are invariant to all seven independent transformations here considered are each apolar to all cubics of the syzygetic sheaf, and to seven of the eight curves of order 3 discussed in $\$ 4$.

This statement would enable us to write readily the equations of these eight line-cubics; but the further remark that all combinants of the syzygetic sheaf are combinants also of the conjugate sheaf of curves of class 3 , and vice versa, while the inflexional triangles play the same rôle in both sheaves, leads to this theorem:

' ' hose curves of class 3 which are invariant to every transformation of cubic line-loci whose symbol is a rational covariant of the fundamental cubic are a sheaf of line-cubics conjugate or doubly apolar to the corresponding members of the syzygetic sheaf of point-cubics, and, further, the eight curves of class 3 which are simultaneously circumscribed and inscribed, each to three out of the four inflexional triangles. Each of these eight line-cubics has the vertices of the fourth inflexional triangle for its Hessian and touches in nine points that one of the eight pointcubics to which it is not apolar.

NoRTh WESTER UnIVERSITY, Evanston, ILL. 